RASĀYAN J. Chem.

Vol. 13 | No. 3 |1498-1505| July - September | 2020 ISSN: 0974-1496 | e-ISSN: 0976-0083 | CODEN: RJCABP

\title{
COMPUTATIONAL APPROACH TO STUDY MARINE DERIVED CORTISTATIN A MOLECULAR MECHANISM AS A JANUS KINASE 3 INHIBITOR
}

\author{
H. Jemmy Christy" and Shiva Shankari \\ Department of Bioinformatics, Sathyabama Institute of Science and Technology, \\ Chennai,600119, India \\ *E-mail: jemmyjacob@gmail.com
}

\begin{abstract}
Cortistatin A is a natural product isolated from the marine sponge Corticium simplex has been considered as a therapeutic agent for autoimmune disorders, HIV infection as well as cancer. Janus kinases (JAKs) and signal transducer and activator of transcription (STAT) transcription factors play a promising role in either causing or participating in the tumorigenesis. Following our earlier studies on JAK-STAT pathway genes varied expression among the various types of breast cancer samples, JAK3 protein plays a crucial role in cancer pathway, thus we implement a feasible systems pharmacology method to explore JAK3 function. Our systematic Insilco approaches involve systems-based assessment of JAK3 role in cytokine signaling, ADMET a scale for pharmacokinetic and pharmacodynamics assessment, pharmacophore mapping of JAK3 and ligand Cortistatin A. Sequence level annotation of functional domains revealed similarity between the JAK family members namely JAK1, JAK2, JAK 3 and TYK2. Thus intermolecular interaction, molecular dynamics and simulation assessment of JAK3 with Cortistatin docked complex pattern applicable other members that shared the domain similarity. Thus, our present study proposes the system level of understanding about Cortistatin A agonist activity on JAK3 and suggesting the compound as a potent antiproliferative agent for cancer treatment.

Keywords: Cortistatin A, JAK Inhibitors, Janus Kinase 3, Inflammation, Cancer Therapy, Kinase Inhibitors.
\end{abstract}

(C) RASĀYAN. All rights reserved

\section{INTRODUCTION}

Signal transduction from cell membrane receptors to the internal nucleus is mediated by the Janus kinase signal transducer and activator of transcription (JAK-STAT) pathway ${ }^{1}$. This promising pathway is a key player in the hematological system regulation as well as immune regulation, thus the pathway genes are the major module of cytokine signalling ${ }^{2}$. In humans, JAK protein family comprised four members namely JAK1, JAK2, JAK3 and TYK2. JAK family proteins embedded with two kinase domains namely JH1 which involved in phosphorylation whereas JH2 involved in JH1 regulation ${ }^{3}$. Deregulation of JAK/STAT pathway reported in various malignant diseases and abnormal immunological response related disorders ${ }^{4}$. Abnormal JAK2 activation causes the $B C R-A B L 1$ negative myeloproliferative neoplasms (MPNs) and JAK inhibitors were the suggested therapeutic option for treating the deregulated JAK pathway-related disease signals ${ }^{5,6}$. Inflammatory signals were activated by JAK/STAT pathway with the help of cytokines like IL-6, IL-9, IL-10, IL-12/23, IL-22, GM-CSF (granulocyte-macrophage colonystimulating factor) and IFN-c (interferon) $)^{7,8}$. Thus JAK proteins were considered for therapeutic targets.JAK3 mediates essential signaling events in both innate and adaptive immunity and plays a crucial role via its association with IL2R, IL4R, IL7R, IL9R, IL15R and IL21R. JAK3, creates docking sites for STATs proteins and upon phosphorylation of the STATs proteins, it activates gene transcription by forming homo or heterodimers. Signals relayed by the JAK3 protein regulate the growth and maturation of certain types of white blood cells called T-cells, B-cells and natural killer cells, which regulate the immune system. JAK3 belongs to a family of cytoplasmic non-receptor tyrosine kinase, which is intracellularly associated with the interleukin-2 receptor subunit gamma to initiate signaling. Marine-

Rasayan J. Chem., 13(3), 1498-1505(2020)

http://dx.doi.org/10.31788/ RJC.2020.1335746

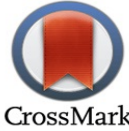


RASĀYAN J. Chem.

Vol. 13 | No. 3 |1498-1505| July - September | 2020

derived natural compounds were known for their diverse chemical structures, therapeutic properties and successful clinical studies suggest the development of marine-derived anticancer drugs ${ }^{9,10}$. Recent studies on anticancer, anti-inflammatory properties of marine-derived compounds include alkaloids, macrolides, terpenoids, among others ${ }^{10}$. A marine steroidal alkaloid Cortistatin A(CA) isolated Study on cortistatin A and other analogs exhibited the effective anti-proliferative against human umbilical vein endothelial cells ${ }^{11}$. Various cancer-specific cell lines namely, KB3-1 (endocervical adenocarcinoma), Neuro2A (neuroblastoma), K562(leukemia) and NHDF(Normal human dermal fibroblast) studies demonstrated the cortistatin antiproliferative activity ${ }^{12}$.JAK3 is mainly restricted to hematopoietic lineage where it plays a vital role in lymphoid cell development and homeostasis ${ }^{13}$. Recent studies have shown that constitutive activation of the JAK-STAT signaling is a characteristic feature of many hematological neoplasms and in various leukemias and lymphomas. The aberrant activation of JAK3 in hematological malignancies suggests JAK3 as a potential target for cancer therapy ${ }^{14}$. Cortistatin A is considered as a potent and selective inhibitor for CDK8 and CDK19 kinases and renders the potent antiangiogenic activity ${ }^{15}$ the computational approach was used to screen their role as JAK inhibitors using molecular docking approach.

\section{EXPERIMENTAL}

\section{Therapeutic Target Protein Jak3 Preparation and Pharmacophore Evaluation}

Tyrosine-protein kinase (JAK3) of sequence length 1124 has been taken from RCSB PDB ID: 4Z16, containing A, B, C, D chains and domains in the region around 800 to 1124 is obtained in pdb format ${ }^{16}$. The total number of binding sites observed is 32 using an eraser algorithm. This has been performed by applying force field CHARMM (Chemistry at Harvard Macromolecular Mechanics) algorithm to study the energetics, refinement and calculation of protein-ligand interaction energies, physics-based scoring of ligands in protein actives and flexibility of molecules ${ }^{17}$ Force fields are used to rank "ligand poses" obtained by a docking algorithm to suggest placement of fragments in sites in the enzyme with the highest binding affinity ${ }^{18}$. Also, the pharmacophore features of the protein been identified using Cavity plus, it is a web server, been used to detect the protein cavity and its associated features using Cav Pharmer to determine pharmacophore modeling, which represents the H-bond donor, H-bond acceptor centers, hydrophobic center, positive electrostatic center, and negative electrostatic center by different colors $^{19}$ Similarly, allosteric site can be identified by CorrSite, where the score should be less than 0.5 which means the cavity may be potential allosteric site ${ }^{20}$. The druggable cysteine residues by CovCys and covalent ligand binding ability using the algorithm CAVITY to detect the potential binding sites on the surface of the given protein structure.

\section{Ligand-Cortistatin A pharmacokinetic Property Evaluation}

The 3D structure of cortistatin A is obtained from a Chemical book in Mol2 format or SDF format from PubChem database ${ }^{21}$.To identify quality lead compounds that show drug-likeness, high throughput screening methods have been used, such as ADMET . The early assessment of the compound can be calculated based on Lipinski's rule of five by predicting absorption, distribution, metabolism, excretion and toxicity (ADMET) properties, which helps to reduce the compounds with unfavorable ADMET characteristics such as Blood-brain barrier penetration, Hepatotoxicity, plasma protein binding, and cytochrome P450 2D6 inhibition. The compound is selected that meets the rules specified by the set of selected SMARTS rules ${ }^{22}$. Similarly, the common pharmacophore features for cortistatin A has been identified to show strong drug-like features of the compound for the structure-based design using Discovery studio- pharmacophore protocol. Using Ultrafast Shape Recognition- Virtual Screening (USRVS tool), the analogs of the ligand have been obtained. This tool aims in identifying the molecules with a similar activity profile across phenotypic and macromolecular targets of previously found molecules to that of a query molecule used as search template ${ }^{23}$. The top 100 similar molecules are obtained compound IDs, similarity scores physicochemical properties, and SMILES. Finally, the pharmacophore mapping and clustering have been performed to validate the ligand using parameters such as hydrogen bond donor, acceptor and hydrophobic regions. This shows that the analogs can also serve as an alternative to the cortistatin A ligand for the drug discovery process ${ }^{24}$. 
RASĀYAN J. Chem.

Vol. 13 | No. 3 |1498-1505| July - September | 2020

\section{Molecular Docking Based Evaluation of Jak3 with Cortistatin A}

The receptor-ligand interaction uses Ligand Fit protocol ${ }^{25}$, which docks the ligands into the binding site of receptors using shape-based searching and Monte Carlo sampling of ligands. The parameters used are PLP1 algorithm for the energy grid and the conjugate gradient for energy minimization. The scores for docked poses are obtained by LigScore1, LigScore ${ }^{26}$ which predicts the binding affinities, -PLP1, -PLP2 known as Piecewise Linear Potential scoring function calculates both the shape and hydrogen bond complementarity of poses to the active site and Jain scoring function which scores the non-covalent protein-ligand interactions ${ }^{27}$.

\section{Molecular Dynamics and Simulation Studies of Jak3 and Cortistatin A Complex}

Molecular dynamics and simulation successfully explore the stability of the docked complex as stable Interaction between the ligand as well as the molecule of the receptor could render the antagonistic activity on the target.

We examined the structural integrity of Jak3 and Cortistatin A in the explicit solvent setting. Molecular dynamics and simulation studies performed using a standard discovery studio cascade dynamics module at v2.1. Using CHARMM forcefield, model KLF6 structure has been applied to assign the position of both the atom and residue and to evaluate the missing atoms using Momany Rone's method of calculating potential levels. Before the production step attempting to allow the complex to evolve spontaneously for some time as well as integrating the equations of motion until the average temperature and structure remained stable, and the total energy converged particle algorithm Mesh Ewald was used to evaluate the long-range electrostatic interactions. The hydrogen assessment was premised on the shake algorithm. ${ }^{28}$ Docked Jak3 and Cortistatin A complex were addressed with a $0.145 \mathrm{~mol} / \mathrm{L} \mathrm{NaCl}$ concentration in a pre-balanced orthorhombic box of TIP3P water. The docked complex was applied under periodic boundary conditions, trying to minimize the system for 2000 steps based on the mechanism of conjugating gradient as well as the steepest descent method. Minimized complex subjected to standard cascade dynamics phases namely heating, equilibration, and production in sequence. Simulation carried out for five ns and temperature of the simulation system was up to 300k and keeping the KLF6 and cinnamic acid complex with a harmonic force constant of $0 \mathrm{kcal} / \mathrm{mol} / \AA 2$. Further equilibration step was performed for 400 PS with a constant pressure of $1 \mathrm{~atm}$. Finally, without any constraints, the production step was performed for five ns at a constant temperature of $300 \mathrm{~K}$ and a constant pressure of $1 \mathrm{~atm} \cdot{ }^{29} \mathrm{The}$ obtained trajectory snapshots of Jak3 and Cortistatin A complex was saved every 2 ps for further analysis.

\section{RESULTS AND DISCUSSION}

Similarly, the chains of JAK1, JAK2 and JAK3 were compared through multiple sequence alignment using Clustal Omega for similar patterns in the amino acid positions 800-1152 (Fig.-1). Cortistatin interacting positions Arg 953, Phe 833, Lys 855, Leu 857, Leu 970 were conserved among JAK1, JAK2 and JAK3. Positions like Glu 871 was conserved between JAK2 and JAK3, but secondary structure propensity was like all three JAK structures. Similarly, Gln 864 position also substituted by His, but structural propensity is alike. The retrieved structure of JAK3 from PDB has structural weight 145875.42. This protein has 4 chains namely A, B, C and D and has domains in the amino acid ranging from 8111124 residue position. The compound has screened for the binding sites prediction and its properties such as druggability, allosteric sites have been shown in (Fig.-2). This shows that the JAK3 has a unique cysteine residue present in the ATP binding pocket of the JAK3 at position 909, which forms covalent binding with the ligand Cortistatin A, which makes it therapeutically active compound for cancer.

Jak3 binding pockets were embedded with ten druggable cavities and their predicted pharmacokinetics score was randing from 6.67 to 6.62.Cumulative drug score ranging from 2545 to 665. Cavity Score and Cavity Drug Score were used to examine and rank the cavities ligandability and druggability of the Jak3 binding cavity. The ligandability value represents the possibility of designing small ligands with high binding affinities to a certain cavity, and the Druggability value reflects the possibility of a cavity being a good target for binding drug-like molecules. Certain cavities were populated with residues that favors high binding affinity to the specified cortistatin A and were represented with high ligandability and druggability scores. In general cavity score of Jak3 calculated based other parameters like cavity volume, 
pocket-size, hydrophobic volume, cavity surface area and hydrogen bond-forming surface area. The CAVITY algorithm retrieved the potential ligand-binding sites of the Jak3 protein surfaces.

Pharmacophore feature screening carried out to retrieve the conformation with the highest fit value (i.e., best fitting the pharmacophore) was assumed as the bioactive conformation for the compound. Cortistatin A pharmacophore screening generated the best conformer with a fit value of 4.99959 .

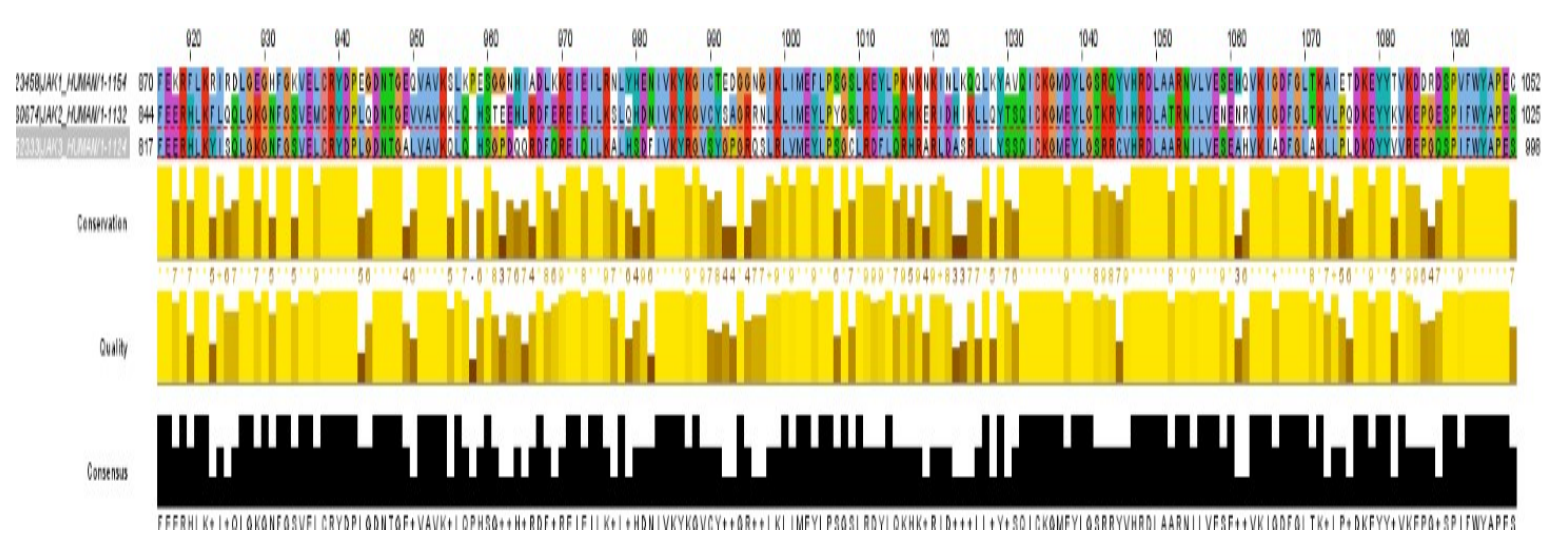

Fig.-1: JAK1,JAK2 and JAK3 Consensus Residues at the Positions Arg 953, Phe 833, Lys 855, Leu 857, Leu 970 highlighted in the JalView Version 2.11.1.0

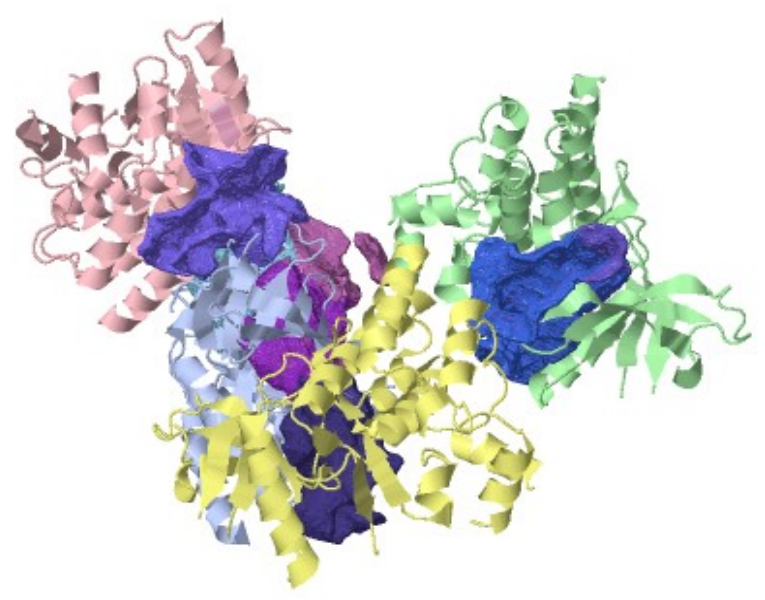

Fig.-2: Druggable Cavities of JAK3 Receptor

(Ten cavities and a covalent-binding site of the Jak3(PDB ID:4Z16) shown in JSmol Visualizer)

ADMET properties are represented in the (Fig.-3a and b) which indicated that the ligand can be used as a therapeutic product. Understanding ADME/Tox is critical for all drug researchers, owing to its increasing importance in advancing high-quality candidates to clinical studies and the processes of drug discovery. The obtained hepatotoxicity probability is 0.258 , PPB level is 0, Absorption level is 0, CYP2D6 probability is estimated as 0.475 and BBB level is 2 . The optimal level of hepatotoxicity of a drug is 0 , which means non-toxic, the BBB levels such as $0,1,2$, and 3 represent a very high, high, moderate and low penetration. Similarly, the absorption level of the drug should be high or medium, that is the value should be either a 0 or 1 so that the drug is absorbed by the intestines after oral administration for further metabolism. The PPB level is also known as plasma protein binding level of drugs which estimates the binding capacity of the drug to the plasma membrane based on the atom-based logarithmic partition coefficient. The values are 0 -Binding $<90 \%$, 1-Binding $>=90 \%, 2$-Binding $>95 \%$.

The Dock-score values include LigScore1, LigScore2, -PLP1, -PLP2, Jain obtained using the ligand fit protocol in Discovery Studio (Fig.-4). The dock-score values for the compound Cortistatin A (ligand) identified from marine sponges with JAK3 are listed in (Fig.-5). The highest dock score obtained from Cortistatin A and JAK3 are 65.804 with 13 hydrogen bonds with the target protein. The binding sites 
RASĀYAN J. Chem.

Vol. 13 | No. 3 |1498-1505| July - September | 2020

show similar amino acids in the same positions, having 13 hydrogen bonds having positive amino acids such as arginine, and lysine (7 carbon-hydrogen bonds and 6 conventional hydrogen bonds) which increases the stability of the interactions (Table-1 and 2).
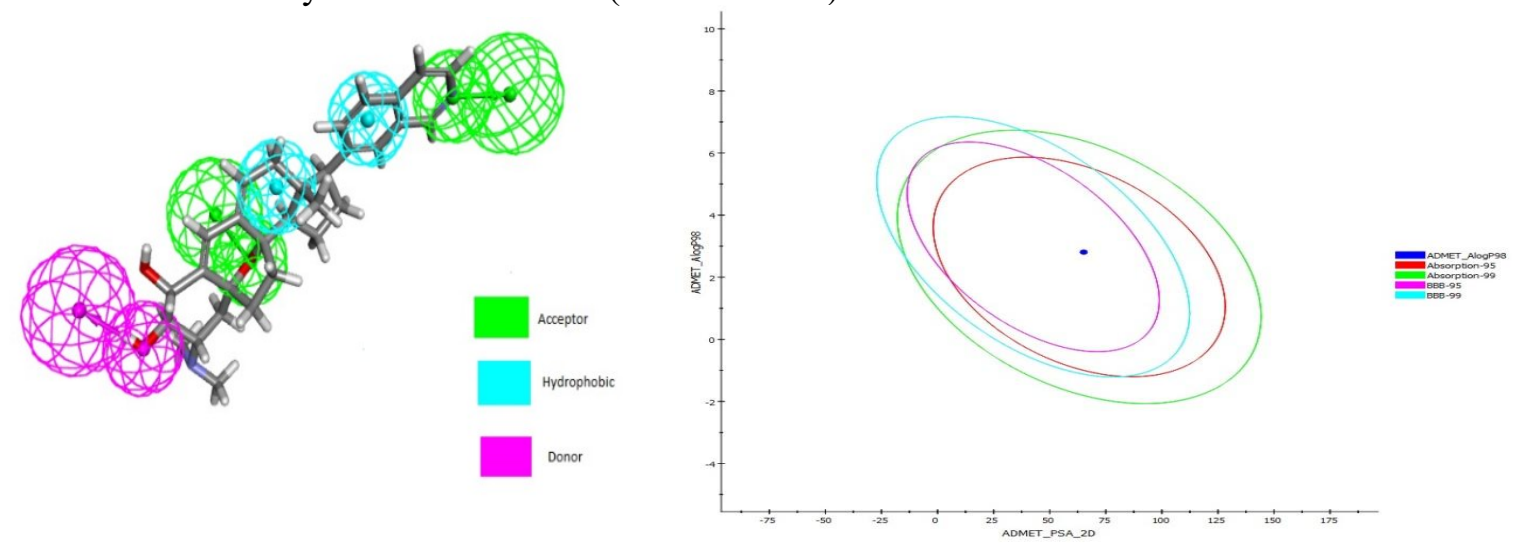

Fig.-3: (a)The common pharmacophore features of Cortistain A were represented with hydrogen bond acceptor and donor feature and hydrophobicity with the highlighted colors green, pink and blue respectively. (b)ADMET:

Prediction of drug absorption evaluation of Cortistatin A. ADMET Descriptors, 2D polar surface area (PSA_2D) in for each compound is plotted against their corresponding calculated atom-type partition coefficient (ALogP98). The area encompassed by the ellipse is a prediction of good absorption with no violation of ADMET properties.

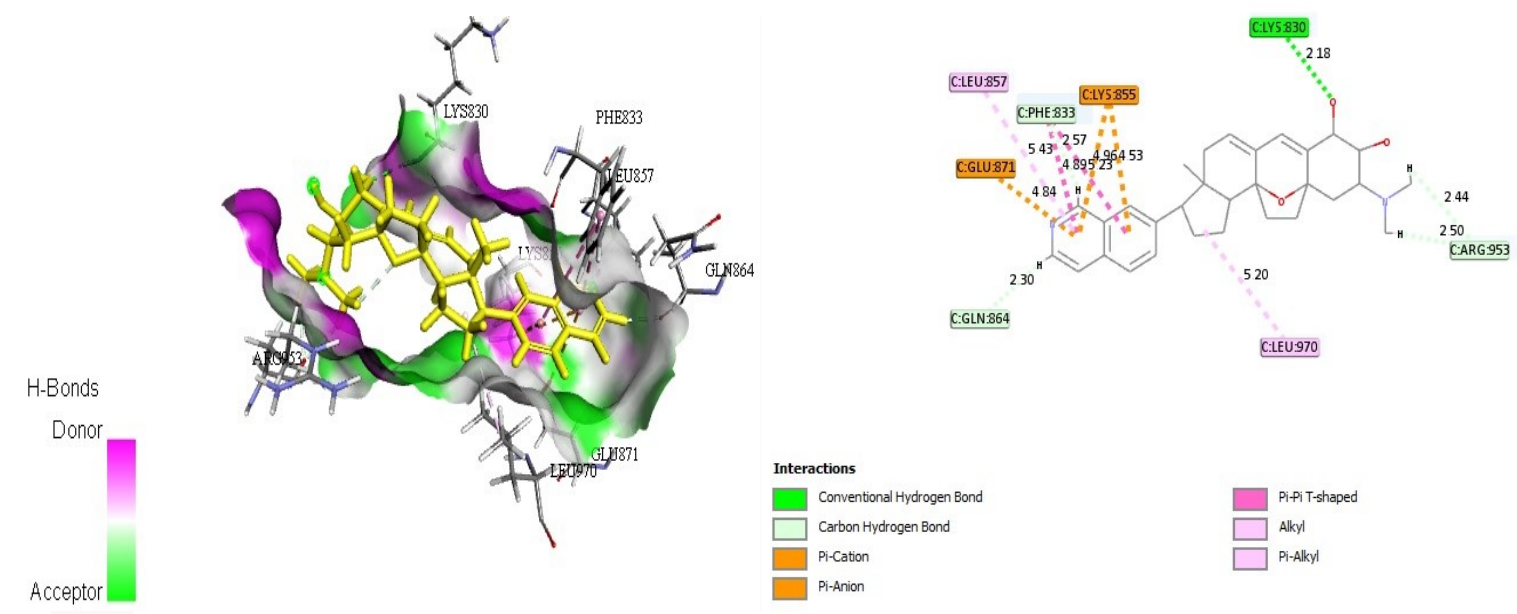

Fig.-4: Docking pose of Cortistatin A in the Binding Cavity of JAK3 Protein Molecule. Right Panel showed the Two-dimensional Plot of the Intermolecular Interactions

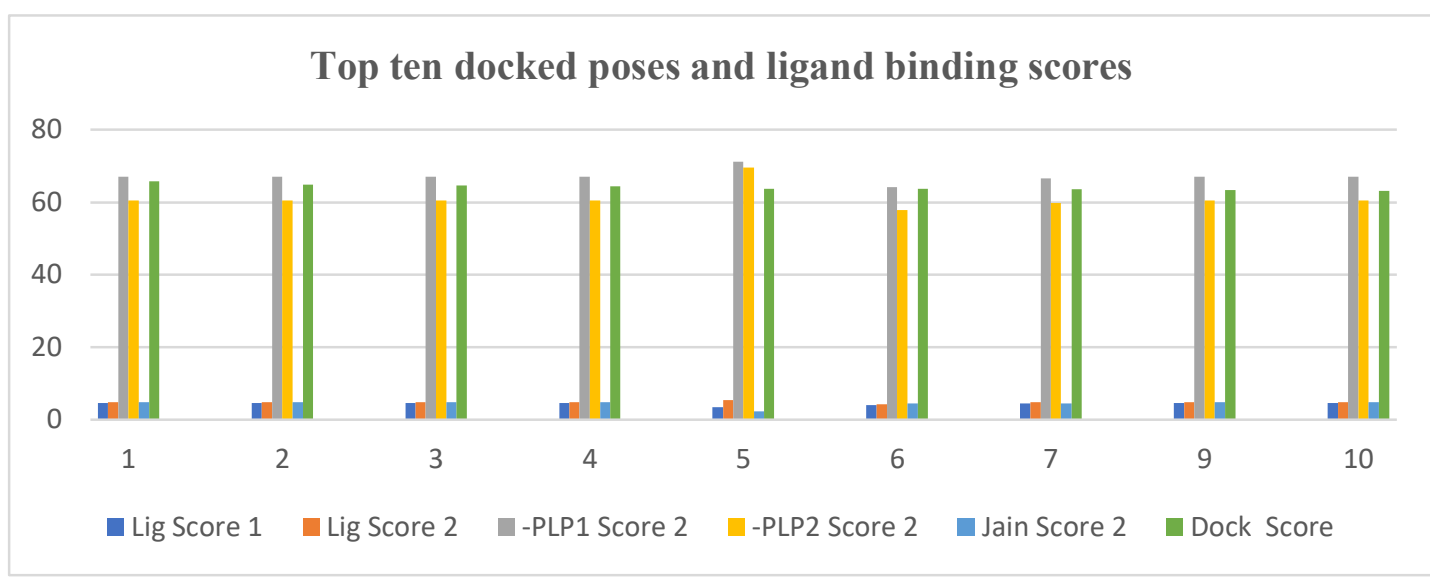

Fig.-5: Dock Score adapted from Ligand Fit as Evaluation Score to Rank the Best Docking Pose of Jak3 and Cortistatin A 
RASĀYAN J. Chem.

Vol. 13 | No. 3 |1498-1505| July - September | 2020

Further Jak3 and cortistatin A docked complexes were subjected to a Molecular Dynamics simulation for a period of 5ns. Energy profiles of the docked complex were listed in Table-2 and Fig.-6.Initial and final potential energy, kinetic and total energy profile were listed as a graph and it was observed that stable energy level was achieved around $-32250 \mathrm{kcal} / \mathrm{mol}$. Simulation and dynamics-based evaluation revealed that intermolecular stability was attained after $3 \mathrm{~ns}$.

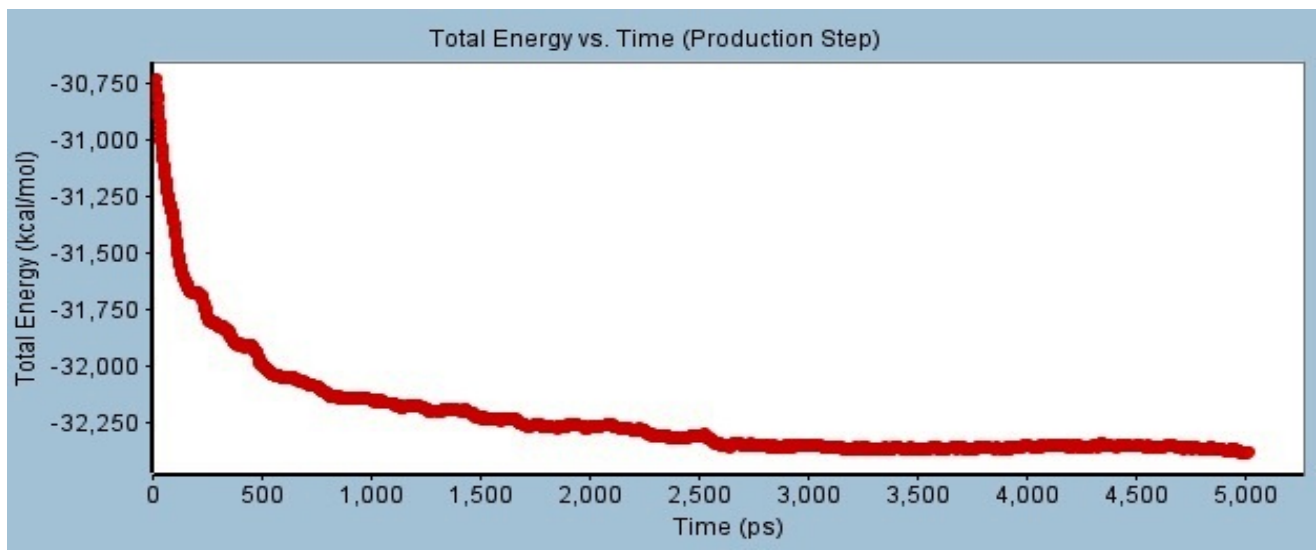

Fig.-6: Total Profile of Jak3 and Cortistatin A Complex Dynamics Simulation Time of 5ns

Table-1: Cortistatin A and Jak3 Protein-interacting Residues that confer conventional Hydrogen Hydrogen Bonds, Carbon Hydrogen Hydrogen bonds, pi-pi ,pi-anion ,pi-cation, Alkyl Bonds.

\begin{tabular}{|c|c|c|c|c|}
\hline Name & Distance & Type of interaction & Donor & Acceptor \\
\hline $\begin{array}{c}\text { Jak3:LYS830:HN - } \\
\text { cortistatin A:O2 }\end{array}$ & 2.17815 & $\begin{array}{c}\text { Conventional } \\
\text { Hydrogen Bond }\end{array}$ & $\begin{array}{c}\text { Jak3:LYS830:HN } \\
\text { - cortistatin } \\
\text { A:O2(Jak1 } \\
\text { Varies) }\end{array}$ & 2.17815 \\
\hline $\begin{array}{c}\text { cortistatin A:H62 - } \\
\text { Jak3:ARG953:O }\end{array}$ & 2.44213 & $\begin{array}{c}\text { Carbon Hydrogen } \\
\text { Bond }\end{array}$ & $\begin{array}{l}\text { cortistatin A:H62 } \\
\text { - Jak3:ARG953:O }\end{array}$ & 2.44213 \\
\hline $\begin{array}{c}\text { cortistatin A:H65 - } \\
\text { Jak3:ARG953:O }\end{array}$ & 2.5018 & $\begin{array}{c}\text { Carbon Hydrogen } \\
\text { Bond }\end{array}$ & $\begin{array}{l}\text { cortistatin A:H65 } \\
\text { - Jak3:ARG953:O }\end{array}$ & 2.5018 \\
\hline $\begin{array}{c}\text { cortistatin A:H69 - } \\
\text { Jak3:PHE833:O }\end{array}$ & 2.56642 & $\begin{array}{c}\text { Carbon Hydrogen } \\
\text { Bond }\end{array}$ & $\begin{array}{l}\text { cortistatin A:H69 } \\
\text { - Jak3:PHE833:O }\end{array}$ & 2.56642 \\
\hline $\begin{array}{l}\text { cortistatin A:H71 - } \\
\text { Jak3:GLN864:O }\end{array}$ & 2.29889 & $\begin{array}{l}\text { Carbon Hydrogen } \\
\text { Bond }\end{array}$ & $\begin{array}{c}\text { cortistatin A:H71 } \\
- \\
\text { Jak3:GLN864:O(J } \\
\text { AK1 AND 2 His) }\end{array}$ & 2.29889 \\
\hline $\begin{array}{c}\text { Jak3:LYS855:NZ - } \\
\text { cortistatin A }\end{array}$ & 4.95987 & Pi-Cation & Jak3:LYS855:NZ & cortistatin A \\
\hline $\begin{array}{c}\text { Jak3:LYS855:NZ - } \\
\text { cortistatin A } \\
\end{array}$ & 4.53399 & Pi-Cation & Jak3:LYS855:NZ & cortistatin A \\
\hline $\begin{array}{c}\text { Jak3:GLU871: } \\
\text { OE1 - cortistatin A }\end{array}$ & 4.83609 & Pi-Anion & $\begin{array}{c}\text { Jak3:GLU871: } \\
\text { OE1(2,3 but } 1 \\
\text { Got Lys) }\end{array}$ & cortistatin $\mathrm{A}$ \\
\hline $\begin{array}{l}\text { cortistatin A - } \\
\text { Jak3:PHE833 }\end{array}$ & 4.89198 & Pi-Pi T-shaped & cortistatin A & Jak3:PHE833 \\
\hline $\begin{array}{l}\text { cortistatin A - } \\
\text { Jak3:PHE833 }\end{array}$ & 5.2272 & Pi-Pi T-shaped & cortistatin A & Jak3:PHE833 \\
\hline $\begin{array}{l}\text { cortistatin A - } \\
\text { Jak3:LEU970 }\end{array}$ & 5.20237 & Alkyl & cortistatin $\mathrm{A}$ & Jak3:LEU970 \\
\hline $\begin{array}{l}\text { cortistatin A - } \\
\text { Jak3:LEU857 }\end{array}$ & 5.43381 & Pi-Alkyl & cortistatin A & Jak3:LEU857 \\
\hline
\end{tabular}


RASĀYAN J. Chem.

Vol. 13 | No. 3 |1498-1505| July - September | 2020

Table-2: Dynamics simulation of Cortistatin A and Jak3 Complex

\begin{tabular}{|c|c|c|c|c|c|c|c|c|c|}
\hline 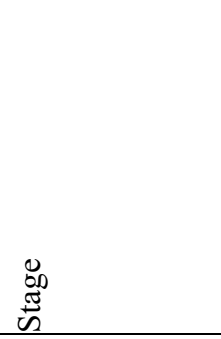 & 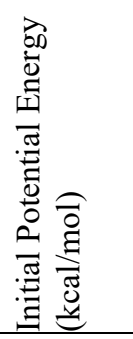 & 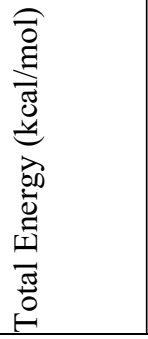 & 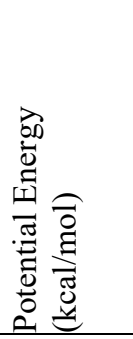 & 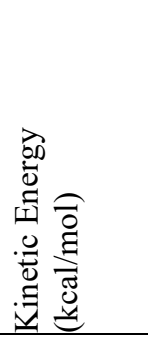 & 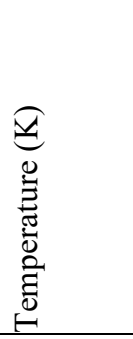 & 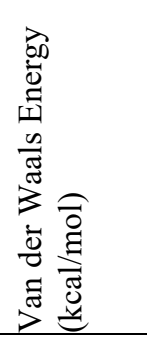 & 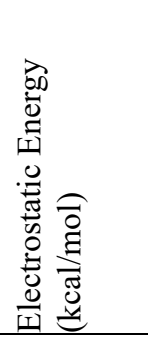 & 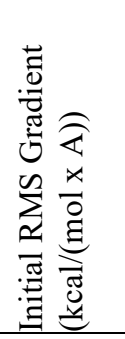 & 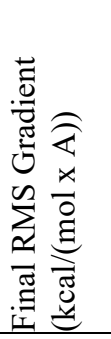 \\
\hline Minimization & -25290.8 & & -34339.3 & & & -4724.85 & -33640.7 & 39.572 & 0.986 \\
\hline Minimization2 & -34339.3 & & -43406.9 & & & -4375.19 & -44046.4 & 0.986 & 0.078 \\
\hline Heating & -43406.9 & -29099.7 & -36670.1 & 7570.369 & 296.754 & -3784.84 & -44005.7 & 3.29 & 19.452 \\
\hline Equilibration & -36670.1 & -30724.8 & -38423.8 & 7698.946 & 301.794 & -3857.37 & -45831.6 & 19.452 & 19.174 \\
\hline Production & -38423.8 & -32377.2 & -40004 & 7626.825 & 298.967 & -3972.69 & -47362.9 & 19.174 & 19.502 \\
\hline
\end{tabular}

\section{CONCLUSION}

The compound and the ligands are studied for pharmacophore activity. The docking studies of the compound were carried out and the results of such studies were reported. In silico studies based on molecular docking and molecular dynamics revealed that the binding sites show moderate dock score of 65.804, having 13 hydrogen bonds having positive amino acids such as arginine, and lysine (7 carbonhydrogen bonds and 6 conventional hydrogen bonds) which increases the stability of the interactions. Similarly, the cysteine residue at position 909 of JAK3 is uniquely present in the ATP binding pocket, which forms covalent binding with the ligand Cortistatin A, which makes it therapeutically active compound for cancer. Having compounds that precisely hit a specific target, like cortistatin A can help reduce side-effects and increase efficacy. Similarly, through Prosite analysis, it has been observed that JAK1, JAK2, and JAK3 shares similar domains and functional groups residues. This indicates that further therapeutic studies can be performed in JAK1 and JAK2 for cancer.

\section{ACKNOWLEDGMENT}

The authors would like to thank the management and technical staff of the Centre for, Molecular Data Science and Systems Biology, Sathyabama Institute of Science, and technology for all the supports and facilities.

\section{REFERENCES}

1. H. Afaf, Al-Nadaf., A Sajeda, Salahb, O. Mutasem. Taha, Computational Biology and Chemistry,74, $263 \mathrm{e} 272(2018)$.

2. J-C Renauld, Nature Reviews Immunology, 3, 667(2003), DOI:10.1038/nri1153

3. J. Christy, L. Priyadharshini, Meta Gene, 16, 122(2018), DOI:10.1016/j.mgene.2018.02.008

4. G. R. Stark, I. M. Kerr, B. R. Williams, R. H. Silverman, R. D. Schreiber, Annual Review of Biochemistry, 67 (1998), DOI:10.1146/annurev.biochem.67.1.227

5. W. Vainchenker, S. N. Constantinescu, Oncogene, 32,21(2013), DOI:10.1038/onc.2012.347

6. R. Rampal, F. Al-Shahrour, O. Abdel-Wahab, Blood, 123, 22(2014), DOI:10.1182/blood-2014-02554634

7. J. Christy, International Journal of Pharma and Biosciences, 4(4),504(2013).

8. M. Flamant, J. Rigaill, S. Paul, X. Roblin, Drugs, 77, 1057(2017), DOI:10.1007/s40265-017-0755-8

9. S. Banerjee, A. Biehl, M. Gadina, S. Hasni, D. M. Schwartz, Current and Future Prospects Drugs, 77,5(2017), DOI: 10.1007/s40265-017-0701-9

10. H. Malve, The Journal of Pharmacy and Bioallied Sciences 8, 83(2016), DOI:10.4103/09757406.171700

11. H. Jemmy Christy, V. Swetha, Asian Journal of Pharmaceutical and Clinical Research, 12, 7(2019), DOI:10.22159/ajpcr.2019.v12i7.33406

12. M. Schumacher, Kelkel, M. Dicato, M. Diederich, Biotechnology Advances, 29, 531(2011), 1504 
RASĀYAN J. Chem.

Vol. 13 | No. 3 |1498-1505| July - September | 2020

DOI: 10.1016/j.biotechadv.2011.02.002

13. S. Aoki, Y. Watanabe, D. Tanabe, M. Arai, H. Suna, K. Miyamoto, H. Tsujibo, K. Tsujikawa, H. Yamamoto, M. Kobayashi, Bioorganic \& Medicinal Chemistry, 15, 6758(2007), DOI: 10.1021 ja902601e

14. Santhanam Vijayasri, Waheeta Hopper, Journal of Applied Pharmaceutical Science, 8 ,01(2018), DOI: 10.7324/JAPS.2018.8104

15. M. L. Nairismägi, M. E. Gerritsen, Z. M. Li, G. C. Wijaya, B. K. H. Chia, Y. Laurensia, J. Q. Lim, X. Yeoh, K.W. S. Yao, W. L. Pang, A. Bisconte, R. J. Hill, J. M. Bradshaw, D. Huang, T. L. L. Song, C. C. Y. Ng, V. Rajasegaran, T. Tang, Q. Q. Tang, X. J. Xia, T. B. Kang, B. T. The, S. T. Lim, C. K. Ong and J. Tan, Leukemia ,32, 1147(2018), DOI:10.1038/s41375-017-0004-x

16. S. J. Thomas, J. A. Snowden, M. P. Zeidler and S. J. Danson, British Journal of Cancer,113, 365(2015), DOI: $10.1038 /$ bjc.2015.233

17. L. Tan, K. Akahane, R. McNally, Journal of Medicinal Chemistry, 58(16), 6589(2015), DOI: 10.1021 acs. jmedchem.5b00710

18. B. R. Brooks, R. E. Bruccoleri, B. D. Olafson, D. J. States, S. Swaminathan and M. Karplus, Journal of Computational Chemistry, 4(2),187(1983), DOI:10.1002/jcc.540040211

19. H. Gohlke, M. Hendlich, G. Klebe, Journal of Molecular Biology, 295, 2(2000), DOI: 10.1006/jmbi.1999.3371

20. Youjun Xu, Shiwei Wang, Qiwan Hu, Shuaishi Gao, Xiaomin Ma, Weilin Zhang, Yihang Shen, Fangjin Chen, Luhua Lai, Jianfeng Pei, Nucleic Acids Research, 46, 2(2018), DOI: $10.1093 /$ nar/gky380

21. X. Ma, H. Meng, L. Lai, The Journal of Chemical Information and Modeling, 56, 1725(2016), DOI: $10.1021 /$ acs.jcim.6b00039

22. S. Kim, J. Chen, T. Cheng, A. Gindulyte, J. He, S. He, Q. Li, B. A. Shoemaker, P. A. Thiessen, B. Yu, L. Zaslavsky, J. Zhang, E. E. Bolton, Nucleic Acids Research, 8, 47(D1), (2019), DOI:10.1093/nar/gky1033

23. S. J. Enoch, J. C. Madden, M.T.D.Cronin, SAR and QSAR in Environmental Research, 19,555 (2008), DOI: 10.1080/10629360802348985

24. P. J. Ballester, W. G. Richards, The Journal of Computational Chemistry, 28, 10, (2007), DOI: $10.1002 /$ jcc. 20681

25. C. M. Venkatachalam, X. Jiang, T. Oldfield, M. Waldman, The Journal of Molecular Graphics and Modelling, 21, 289(2003), DOI:10.1016/s1093-3263(02) 00164-X

26. A. Krammer, P. D. Kirchhoff, X. Jiang, C. M. Venkatachalam, M. Waldman, The Journal of Molecular Graphics and Modelling, 23, 395(2005), DOI:10.1016/j.jmgm.2004.11. 007

27. A. N. Jain, Anthony Nicholls, Journal of Computer-Aided Molecular Design,22, 3(2008), DOI: 10.1007/s10822-008-9196-5

28. J. Mortier, C. Rakers, M. Bermudez, M. S. Murgueitio, S. Riniker, G. Wolber, Drug Discovery Today, 20, 686(2015), DOI:10.1016/j.drudis.2015.01.003

29. J. L. Zhang, Q. C. Zheng, H. X. Zhang, The Journal of Physical Chemistry B, 114,7383 (2010), DOI:10.1371/journal.pone.0039546

[RJC-5746/2020] 